\title{
Ancient solutions of superlinear heat equations on Riemannian manifolds
}

\author{
Daniele Castorina * $\quad$ Carlo Mantegazza $^{\dagger}$
}

August 2, 2017

\begin{abstract}
We study some qualitative properties of ancient solutions of superlinear heat equations on a Riemannian manifold, with particular interest in positivity and constancy in space.
\end{abstract}

\section{Introduction}

In this paper we continue the study started in [2] about some properties of solutions of semilinear heat equations on a complete and connected Riemannian manifold $(M, g)$ without boundary. We will consider the model equations $u_{t}=\Delta u+|u|^{p}$ with $p>1$, where $\Delta$ is the Laplace-Beltrami operator of $(M, g)$.

Definition 1.1. We call a solution of the equation $u_{t}=\Delta u+|u|^{p}$

- ancient if it is defined in $M \times(-\infty, T)$ for some $T \in \mathbb{R}$,

- immortal if it is defined in $M \times(T,+\infty)$ for some $T \in \mathbb{R}$,

- eternal if it is defined in $M \times \mathbb{R}$.

We call a solution $u$ trivial if it is constant in space, that is, $u(x, t)=u(t)$ and solves the ODE $u^{\prime}=|u|^{p}$. We say that $u$ is simply constant if it is constant in space and time.

Notice that positive ancient (or negative immortal) trivial solutions always exists (the problem reduces to solve the above ODE).

${ }^{*}$ Dipartimento di Matematica, Università di Roma Tor Vergata, Via della Ricerca Scientifica, 00133 Roma, Italy, castorin@mat.uniroma2.it

${ }^{\dagger}$ Dipartimento di Matematica e Applicazioni, Università di Napoli, Via Cintia, Monte S. Angelo 80126 Napoli, Italy, c.mantegazza@sns.it 
A reason for the interest in ancient or eternal solutions is that they typically arise as blow-up limits when the solutions of semilinear parabolic equations (in bounded intervals) develop a singularity at a certain time $T \in \mathbb{R}$, i.e. the solution $u$ becomes unbounded as $t \rightarrow T^{-}$.

Our main result is the following theorem.

Theorem 1.2. Let $(M, g)$ be a n-dimensional compact Riemannian manifold without boundary such that Ric $>0$. Let $u$ be an ancient solution to the semilinear heat equation $u_{t}=\Delta u+|u|^{p}$, with $1<p<\frac{n(n+2)}{(n-1)^{2}}$. Then $u$ is trivial.

In Theorem 4.4 of [2] we proved triviality of ancient solutions for the case $p=2$ under a suitable growth assumption, hence, Theorem 1.2, besides extending the conclusion to any exponent $1<p<\frac{n(n+2)}{(n-1)^{2}}$, improves such result not requiring any growth assumptions on the solutions. This is achieved through a priori gradient and decay estimates of independent interest, which we discuss in Section 3. Moreover, we underline that we do not assume the positivity of the solutions, but we just obtain it under the hypothesis of boundedness from below of the Ricci tensor (see Section 2), thus, as a consequence, it is possible to improve results such as Theorem 1 in [14] or Corollary 1.6 in [12].

In the Euclidean space, it is well known that nontrivial global radial (static) solutions on $\mathbb{R}^{n} \times \mathbb{R}$ exist for any supercritical exponent $p \geq \frac{n+2}{n-2}$. Conversely, while triviality of eternal radial solutions can be shown in the full range of subcritical exponents $1<p<\frac{n+2}{n-2}$, the same expected result for general (not necessarily radial) solutions is known only in the range $1<p<\frac{n(n+2)}{(n-1)^{2}}$ (as in Theorem 1.2 above), and it remains a challenging open problem when $\frac{n(n+2)}{(n-1)^{2}} \leq p<\frac{n+2}{n-2}$ (see [4,5]).

In Section 2 we show that the boundedness from below of the Ricci tensor of the manifold $(M, g)$ implies the positivity of ancient solutions. In Section 3 we obtain a universal a priori estimate which implies the decay at minus infinity of ancient solutions, as well as a gradient estimate of Li-Yau type. Finally, in Section 4, we prove Theorem 1.2 as a corollary of a triviality result.

In all of the paper, the Riemannian manifolds $(M, g)$ will be smooth, complete, connected and without boundary. We will denote with $\Delta$ the associated Laplace-Beltrami operator. As it is standard, we will write Ric $\geq \lambda$ with the meaning Ric $\geq \lambda g$, that is, all the eigenvalues of the Ricci tensor are larger or equal than $\lambda \in \mathbb{R}$. Finally, all the solutions we will consider are classical, $C^{2}$ in space and $C^{1}$ in time.

\section{Positivity}

We start showing positivity of ancient solutions. We will also see that eternal solutions are trivial in the compact case.

Proposition 2.1. Let $(M, g)$ be compact and $u$ an ancient solution of the equation $u_{t}=\Delta u+|u|^{p}$ with $p>1$ in $M \times(-\infty, T)$, for some $T \in \mathbb{R}$, then either $u \equiv 0$ or $u>0$ everywhere. 
Proof. For every $t<T$, we define $x_{t} \in M$ as the point such that $u\left(x_{t}, t\right)=\min _{x \in M} u(x, t)$ and we set $v(t)=u\left(x_{t}, t\right)$, then, by maximum principle or more precisely, by Hamilton's trick (see [8] or [10, Lemma 2.1.3] for details), at almost every $t \in(-\infty, T)$ (precisely when $v^{\prime}(t)$ exists - notice that $v$ is locally Lipschitz, as $M$ is compact) there holds $v^{\prime}(t) \geq|v(t)|^{p}$.

If $v\left(t_{0}\right)<0$ at some time $t_{0} \in(-\infty, T)$, then integrating the above differential inequality in intervals $\left[t_{1}, t_{0}\right]$, it is easy to see that, moving in the past, $v(t)$ goes to $-\infty$ in finite time, which is a contradiction. Thus, $v(t) \geq 0$ for every $t \in(-\infty, T)$, which implies $u \geq 0$ everywhere. Then, by strong maximum principle, either $u>0$ everywhere ( $M$ is connected) or $u \equiv 0$ and we are done.

Corollary 2.2. If $(M, g)$ is compact and $u$ is an eternal solution of $u_{t}=\Delta u+|u|^{p}$ with $p>1$ in $M \times \mathbb{R}$, then $u \equiv 0$.

Proof. By the previous proposition, if $u \not \equiv 0$, then $u$ is positive everywhere and (with the same notation of the previous theorem) if $v\left(t_{0}\right)>0$, for some $t_{0} \in \mathbb{R}$, by integrating the differential inequality $v^{\prime}(t) \geq v^{p}(t)$, we see that $v(t)$ goes to $+\infty$ in finite time, against the hypothesis that $u$ is an eternal solution.

Remark 2.3. With the same argument, we can see that an immortal nonnegative solution is identically zero.

Remark 2.4. In the noncompact situation, the conclusion of Corollary 2.2 does not necessarily hold. Consider $M=\mathbb{R}^{n}$ and $u: \mathbb{R}^{n} \rightarrow \mathbb{R}$ given by a "Talenti's function" (an extremal of Sobolev inequality, see [17] and also [1]), that is,

$$
u(x)=\left(\frac{n(n-2)}{n(n-2)+|x|^{2}}\right)^{\frac{n-2}{2}},
$$

which, by a straightforward computation, satisfies $\Delta u+u^{\frac{n+2}{n-2}}=0$ in $\mathbb{R}^{n}$, for $n \geq 3$. In particular, $u$ is a nonzero eternal (static) solution for the semilinear heat equation $u_{t}=$ $\Delta u+|u|^{p}$, with $p=\frac{n+2}{n-2}$.

We deal now with the noncompact case, following the technique of [3, Proposition 2.1].

Lemma 2.5. Let the Ricci tensor of the $n$-dimensional Riemannian manifold $(M, g)$ be uniformly bounded below by $-K(n-1)$, with $K \geq 0$, and let $u$ be a solution of the equation $u_{t}=\Delta u+|u|^{p}$ with $p>1$ in $M \times[0, T)$. For any $0<\delta<1$, there is a constant $C_{\delta}>0$ such that, if $u \geq-L$, for some positive value $L \in \mathbb{R}$, in the ball $B_{A r_{0}}\left(x_{0}\right)$ at $t=0$, with

$$
A \geq 4+2(n-1) T / r_{0}^{2}+2(n-1) T \sqrt{K} / r_{0},
$$

then,

$$
u(x, t) \geq \min \left\{-\left((1-\delta)(p-1) t+L^{1-p}\right)^{\frac{1}{1-p}},-\frac{C_{\delta}}{\left(A r_{0}\right)^{\frac{2}{p-1}}}\right\}
$$

for every $x \in B_{A r_{0} / 4}\left(x_{0}\right)$ and $t \in[0, T)$. 
Proof. By the Laplacian comparison theorem (see [13, Chapter 9, Section 3.3] and also [15]), if Ric $\geq-K(n-1)$, with $K \geq 0$, we have

$$
-\Delta d\left(x_{0}, x\right) \geq-\frac{n-1}{d\left(x_{0}, x\right)}-(n-1) \sqrt{K} \geq-\frac{n-1}{r_{0}}-(n-1) \sqrt{K}
$$

whenever $d\left(x_{0}, x\right) \geq r_{0}$, in the sense of support functions (or in the sense of viscosity, see [6] check also [11, Appendix A] for comparison of the two notions), in particular, this inequality can be used in maximum principle arguments, see again [13, Chapter 9, Section 3], for instance. Hence,

$$
-\Delta d\left(x_{0}, x\right)+\frac{n-1}{r_{0}}+(n-1) \sqrt{K} \geq 0,
$$

for every $x \in M$ such that $d\left(x_{0}, x\right) \geq r_{0}$.

We consider the function $w(x, t)=u(x, t) \psi(x, t)$ with

$$
\psi(x, t)=\varphi\left(\frac{d\left(x_{0}, x\right)+\left(\frac{n-1}{r_{0}}+(n-1) \sqrt{K}\right) t}{A r_{0}}\right),
$$

where $\varphi: \mathbb{R} \rightarrow \mathbb{R}$ is a smooth, nonnegative and nonincreasing function such that $\varphi=1$ in $(-\infty, 3 / 4]$ and $\varphi=0$ in $[1,+\infty)$. Moreover, we ask that $\varphi,-\varphi^{\prime}$ and $\varphi^{\prime \prime}$ are all positive in a small interval $(1-\varepsilon, 1) \subset(3 / 4,1)$.

For every $t \in[0, T)$, we let $w_{\min }(t)=\min _{x \in M} w(x, t)$ which is well defined (by construction, $\varphi(s)$ is zero for $s \geq 1)$. Moreover, $w_{\min }$ is locally Lipschitz, hence differentiable at almost every time $t \in[0, T)$. If $w_{\min }(t)<0$, the minimum of $w(\cdot, t)$ is achieved at some point $x_{t} \in B_{A r_{0}}\left(x_{0}\right)$, with $\psi\left(x_{t}, t\right)>0$ and $u\left(x_{t}, t\right)<0$. Hence, in a space-time neighborhood of $\left(x_{t}, t\right)$ we have $\varphi(\ldots)=\psi>0$, so we can compute

$$
\begin{aligned}
\left(\frac{\partial}{\partial t}-\Delta\right) w= & \psi\left(\frac{\partial}{\partial t}-\Delta\right) u+u\left(\frac{\partial}{\partial t}-\Delta\right) \psi-2 \nabla \psi \nabla u \\
= & \psi|u|^{p}+\varphi^{\prime}(\ldots) \frac{u}{A r_{0}}\left[-\Delta d\left(x_{0}, \cdot\right)+\frac{n-1}{r_{0}}+(n-1) \sqrt{K}\right] \\
& -\varphi^{\prime \prime}(\ldots) \frac{u}{A^{2} r_{0}^{2}}-2 \nabla \psi \nabla u \\
= & \psi|u|^{p}+\varphi^{\prime}(\ldots) \frac{u}{A r_{0}}\left[-\Delta d\left(x_{0}, \cdot\right)+\frac{n-1}{r_{0}}+(n-1) \sqrt{K}\right] \\
& -\varphi^{\prime \prime}(\ldots) \frac{u}{A^{2} r_{0}^{2}}-2 \frac{\nabla \psi \nabla w}{\psi}+2 u \frac{|\nabla \psi|^{2}}{\psi} \\
= & \psi|u|^{p}+\varphi^{\prime}(\ldots) \frac{u}{A r_{0}}\left[-\Delta d\left(x_{0}, \cdot\right)+\frac{n-1}{r_{0}}+(n-1) \sqrt{K}\right] \\
& -2 \frac{\nabla \psi \nabla w}{\psi}+\frac{u}{A^{2} r_{0}^{2}}\left(\frac{2\left[\varphi^{\prime}(\ldots)\right]^{2}}{\varphi(\ldots)}-\varphi^{\prime \prime}(\ldots)\right),
\end{aligned}
$$

at the smooth points of the function $\psi$, in particular, at the smooth points of the distance function (that is, the points not belonging to the cutlocus of $x_{0}$ ). Notice that we used the fact that $\left|\nabla d\left(x_{0}, \cdot\right)\right|^{2}=1$. 
If $x_{t} \in B_{2 r_{0}}\left(x_{0}\right)$, we have

$$
\begin{aligned}
\frac{d\left(x_{0}, x_{t}\right)+\left(\frac{n-1}{r_{0}}+(n-1) \sqrt{K}\right) t}{A r_{0}} & \leq \frac{2 r_{0}+\left(\frac{n-1}{r_{0}}+(n-1) \sqrt{K}\right) T}{A r_{0}} \\
& \leq \frac{2 r_{0}+(n-1) T / r_{0}+(n-1) \sqrt{K} T}{4 r_{0}+2(n-1) T / r_{0}+2(n-1) \sqrt{K} T}=1 / 2,
\end{aligned}
$$

by the hypothesis on $A$.

Thus, by the choice of $\varphi$, the function $\psi$ is locally constant equal to 1 around $\left(x_{t}, t\right)$, hence smooth and $\nabla \psi=\Delta \psi=\frac{\partial \psi}{\partial t}=0$. It follows, by the first line in computation (2.2), that in such case there holds

$$
\left(\frac{\partial}{\partial t}-\Delta\right) w=\psi|u|^{p}
$$

locally around $\left(x_{t}, t\right)$.

If instead $d\left(x_{t}, x_{0}\right) \geq 2 r_{0}$, estimate (2.1) and formula (2.2) hold in sense of support functions (or of viscosity), locally around $\left(x_{t}, t\right)$. Moreover, $\varphi^{\prime}(\ldots) u \geq 0$, as $u$ is locally negative, that is, the factor in front of the second term in the right hand side of formula (2.2) is nonnegative. Hence, locally we have

$$
\begin{aligned}
\left(\frac{\partial}{\partial t}-\Delta\right) w & \geq \psi|u|^{p}-2 \frac{\nabla \psi \nabla w}{\psi}+\frac{u}{A^{2} r_{0}^{2}}\left(\frac{2\left[\varphi^{\prime}(\ldots)\right]^{2}}{\varphi(\ldots)}-\varphi^{\prime \prime}(\ldots)\right) \\
& \geq \psi|u|^{p}-2 \frac{\nabla \psi \nabla w}{\psi}-\frac{|u|}{A^{2} r_{0}^{2}}\left|\frac{2\left[\varphi^{\prime}(\ldots)\right]^{2}}{\varphi(\ldots)}-\varphi^{\prime \prime}(\ldots)\right| .
\end{aligned}
$$

Notice that, by the argument leading to equation (2.3), this conclusion holds also when $x_{t} \in B_{2 r_{0}}\left(x_{0}\right)$, hence, independently of the position of the point $x_{t}$, for every time $t \in[0, T)$ such that $w_{\min }(t)<0$.

Then, by maximum principle (Hamilton's trick) and standard support functions (or viscosity) techniques, if $w_{\min }^{\prime}(t)$ exists, we get the inequality

$$
w_{\min }^{\prime}(t) \geq \varphi(\ldots)|u|^{p}-\frac{|u|}{A^{2} r_{0}^{2}}\left|\frac{2\left[\varphi^{\prime}(\ldots)\right]^{2}}{\varphi(\ldots)}-\varphi^{\prime \prime}(\ldots)\right|,
$$

with the right hand side evaluated at $\left(x_{t}, t\right)$.

Now, it is not difficult to see that, by our assumptions on the function $\varphi: \mathbb{R} \rightarrow \mathbb{R}$, there exists a positive constant $C$ such that $\left|\frac{2\left[\varphi^{\prime}\right]^{2}}{\varphi}-\varphi^{\prime \prime}\right| \leq C \varphi^{1 / p}$, as $p>1$. Hence, simplifying 
the notation, for any $\delta \in(0,1)$, there holds

$$
\begin{aligned}
w_{\min }^{\prime} & \geq \varphi|u|^{p}-\frac{C|u| \varphi^{1 / p}}{A^{2} r_{0}^{2}} \\
& \geq \varphi|u|^{p}-\frac{\delta}{p} \varphi|u|^{p}-\frac{(p-1) C^{\frac{p}{p-1}}}{p \delta^{\frac{1}{p-1}} A^{\frac{2 p}{p-1}} r_{0}^{\frac{2 p}{p-1}}} \\
& =\frac{\psi^{p}|u|^{p}}{\psi^{p-1}}(1-\delta / p)-\frac{C_{p}}{\delta^{\frac{1}{p-1}} A^{\frac{2 p}{p-1}} r_{0}^{\frac{2 p}{p-1}}} \\
& =\frac{\left|w_{\min }\right|^{p}}{\psi^{p-1}}(1-\delta / p)-\frac{C_{p}}{\delta^{\frac{1}{p-1}} A^{\frac{2 p}{p-1}} r_{0}{ }^{\frac{2 p}{p-1}}},
\end{aligned}
$$

where we used Young inequality, for some positive constant $C_{p}$. As $0<\psi\left(x_{t}, t\right) \leq 1$, when $w_{\min }(t)<0$, we get

$$
\begin{aligned}
w_{\text {min }}^{\prime} & \geq\left|w_{\min }\right|^{p}(1-\delta)+\frac{\delta(p-1)}{p}\left|w_{\min }\right|^{p}-\frac{C_{p}}{\delta^{\frac{1}{p-1}} A^{\frac{2 p}{p-1}} r_{0}^{\frac{2 p}{p-1}}} \\
& \geq\left|w_{\min }\right|^{p}(1-\delta)+\frac{\delta(p-1)}{p}\left(\left|w_{\min }\right|^{p}-\frac{C_{\delta}^{p}}{A^{\frac{2 p}{p-1}} r_{0}^{\frac{2 p}{p-1}}}\right)
\end{aligned}
$$

for some positive constant $C_{\delta}$, at almost every time $t \in[0, T)$ such that $w_{\min }(t)<0$.

Resuming, for almost every $t \in[0, T)$, either $w_{\min }(t)$ is nonnegative or the differential inequality (2.4) holds, moreover $w_{\min }(0) \geq-L$, by hypothesis (recall that if $w_{\min }(0)<$ 0 any point of minimum of $w(\cdot, 0)$ belongs to $\left.B_{A r_{0}}\left(x_{0}\right)\right)$. By an easy ODE's argument, integrating such differential inequality, we conclude

$$
w_{\min }(t) \geq \min \left\{-\left((1-\delta)(p-1) t+L^{1-p}\right)^{\frac{1}{1-p}},-\frac{C_{\delta}}{\left(A r_{0}\right)^{\frac{2}{p-1}}}\right\},
$$

for every $t \in[0, T)$. It follows that

$$
u(x, t)=u(x, t) \psi(x, t)=w(x, t) \geq \min \left\{-\left((1-\delta)(p-1) t+L^{1-p}\right)^{\frac{1}{1-p}},-\frac{C_{\delta}}{\left(A r_{0}\right)^{\frac{2}{p-1}}}\right\}
$$

for every $x \in B_{A r_{0} / 4}\left(x_{0}\right)$ and $t \in[0, T)$.

As a consequence of this lemma, we prove the positivity of ancient solutions. Notice that we do not ask any bound on $u$.

Theorem 2.6. Let the Ricci tensor of $(M, g)$ be uniformly bounded below. If $u$ is an ancient solution of the equation $u_{t}=\Delta u+|u|^{p}$ with $p>1$ in $M \times(-\infty, T)$, then either $u \equiv 0$ or $u>0$ everywhere. 
Proof. We only need to show that $u \geq 0$ everywhere, then the conclusion will follow by the strong maximum principle, as before.

Let the Ricci tensor of $(M, g)$ be bounded below by $-K(n-1)$, for some $K \geq 0$. Since the estimate in the previous lemma is invariant by translation in time, for every $m \in \mathbb{N}$, we can consider the interval $[-m, T)$, for any $m>-T$, and conclude that

$$
u(x, t) \geq \min \left\{-\left((1-\delta)(p-1)(t+m)+L^{1-p}\right)^{\frac{1}{1-p}},-\frac{C_{\delta}}{\left(A r_{0}\right)^{\frac{2}{p-1}}}\right\},
$$

for every $x \in B_{A r_{0} / 4}\left(x_{0}\right)$ and $t \in[-m, T)$, with $-L \leq \inf _{x \in B_{A r_{0}}\left(x_{0}\right)} u(x,-m)$ and

$$
A \geq 4+2(n-1)(T+m) / r_{0}^{2}+2(n-1)(T+m) \sqrt{K} / r_{0} .
$$

In particular, for every $t \in[-m+1, T)$ and $x \in B_{A r_{0} / 4}\left(x_{0}\right)$, sending $L$ to $+\infty$, we get

$$
u(x, t) \geq \min \left\{-((1-\delta)(p-1)(t+m))^{\frac{1}{1-p}},-\frac{C_{\delta}}{\left(A r_{0}\right)^{\frac{2}{p-1}}}\right\} .
$$

Sending now $A \rightarrow+\infty$, we have that for every $t \in[-m+1, T)$ and $x \in M$ there holds

$$
u(x, t) \geq-((1-\delta)(p-1)(t+m))^{\frac{1}{1-p}},
$$

for every $m \in \mathbb{N}$, large enough. Sending finally $m \rightarrow+\infty$, we conclude that $u \geq 0$ everywhere.

Corollary 2.7. Let the Ricci tensor of $(M, g)$ be uniformly bounded below. If $u$ is a solution of the semilinear elliptic equation $\Delta u+|u|^{p}=0$ with $p>1$ in $M$, then either $u \equiv 0$ or $u>0$ everywhere.

\section{Gradient and decay estimates}

We now show a gradient estimate for positive solutions of the semilinear heat equation $u_{t}=\Delta u+|u|^{p}$ on manifolds with nonnegative Ricci tensor. A similar result for the classical heat equation has been proven by Souplet and Zhang in [16]. We will then apply this estimate in order to obtain the triviality of ancient solutions of $u_{t}=\Delta u+|u|^{p}$ under some hypotheses.

Lemma 3.1. Let $(M, g)$ be an $n$-dimensional Riemannian manifold with $\mathrm{Ric} \geq K(n-1)$, for some $K \geq 0$ and let $p>1$. Let $u$ be a positive solution of the semilinear heat equation $u_{t}=\Delta u+|u|^{p}$ in $Q_{R, T}=B\left(x_{0}, R\right) \times\left[T_{0}-T, T_{0}\right]$, with $B\left(x_{0}, R\right)$ the geodesic ball centered at $x_{0}$ of radius $R$ in $M$. Assume that $u \leq D$ in $Q_{R, T}$. Then, there exists a constant $C=C(n, p)$ such that on $Q_{R / 2, T / 4}$ there holds

$$
\frac{|\nabla u(x, t)|}{u(x, t)} \leq C\left(\frac{1}{R}+\frac{1}{\sqrt{T}}+\sqrt{\left(p D^{p-1}-(n-1) K\right)_{+}}\right)\left(1+\log \frac{D}{u(x, t)}\right),
$$

where $\left(p D^{p-1}-(n-1) K\right)_{+}$denotes $\max \left\{p D^{p-1}-(n-1) K, 0\right\}$. 
Proof. Let us define

$$
f=\log \left(\frac{u}{D}\right), \quad w=\frac{|\nabla f|^{2}}{(1-f)^{2}} .
$$

Thanks to the semilinear heat equation we easily see that

$$
f_{t}=\Delta f+|\nabla f|^{2}+\left(D e^{f}\right)^{p-1},
$$

then, in an orthonormal basis, we have

$$
\begin{aligned}
w_{t} & =\frac{2 \nabla f \nabla f_{t}}{(1-f)^{2}}+\frac{2|\nabla f|^{2} f_{t}}{(1-f)^{3}} \\
& =\frac{2 \nabla f \nabla\left(\Delta f+|\nabla f|^{2}+\left(D e^{f}\right)^{p-1}\right)}{(1-f)^{2}}+\frac{2|\nabla f|^{2}\left(\Delta f+|\nabla f|^{2}+\left(D e^{f}\right)^{p-1}\right)}{(1-f)^{3}} \\
& =\frac{2 \nabla f \nabla\left(\Delta f+|\nabla f|^{2}\right)}{(1-f)^{2}}+\frac{2|\nabla f|^{2}\left(\Delta f+|\nabla f|^{2}\right)}{(1-f)^{3}}+\frac{2|\nabla f|^{2}\left(D e^{f}\right)^{p-1}}{(1-f)^{2}}\left[(p-1)+\frac{1}{1-f}\right] \\
& =\frac{2 f_{j i i} f_{j}+4 f_{i} f_{j} f_{i j}}{(1-f)^{2}}+\frac{2 f_{i}^{2} f_{j j}+2|\nabla f|^{4}}{(1-f)^{3}}+\frac{2|\nabla f|^{2}\left(D e^{f}\right)^{p-1}}{(1-f)^{2}}\left[(p-1)+\frac{1}{1-f}\right] \\
& =\frac{2 f_{i i j} f_{j}-2 \operatorname{Ric}_{i j} f_{i} f_{j}+4 f_{i} f_{j} f_{i j}}{(1-f)^{2}}+\frac{2 f_{i}^{2} f_{j j}+2|\nabla f|^{4}}{(1-f)^{3}}+\frac{2|\nabla f|^{2}\left(D e^{f}\right)^{p-1}}{(1-f)^{2}}\left[(p-1)+\frac{1}{1-f}\right],
\end{aligned}
$$

where we interchanged derivatives (hence, there is an "extra" error term given by the Ricci tensor), passing from the fourth to the fifth line and we used the usual convention of summing on repeated indexes.

Now,

$$
\nabla_{j} w=\nabla_{j}\left(\frac{f_{i}^{2}}{(1-f)^{2}}\right)=\frac{2 f_{i} f_{j i}}{(1-f)^{2}}+\frac{2 f_{i}^{2} f_{j}}{(1-f)^{3}}
$$

and

$$
\Delta w=\frac{2 f_{i j}^{2}}{(1-f)^{2}}+\frac{2 f_{i} f_{j j i}}{(1-f)^{2}}+\frac{8 f_{i} f_{i j} f_{j}}{(1-f)^{3}}+\frac{2 f_{i}^{2} f_{j j}}{(1-f)^{3}}+\frac{6 f_{i}^{2} f_{j}^{2}}{(1-f)^{4}} .
$$

Hence, we get

$$
\begin{aligned}
w_{t}-\Delta w= & \frac{2 f_{j} f_{i i j}-2 \operatorname{Ric}_{i j} f_{i} f_{j}+4 f_{i} f_{i j} f_{j}}{(1-f)^{2}}+\frac{2 f_{i}^{2} f_{j j}+2|\nabla f|^{4}}{(1-f)^{3}} \\
& +\frac{2|\nabla f|^{2}\left(D e^{f}\right)^{p-1}}{(1-f)^{2}}\left[(p-1)+\frac{1}{1-f}\right] \\
& -\frac{2 f_{i j}^{2}}{(1-f)^{2}}-\frac{2 f_{i} f_{j j i}}{(1-f)^{2}}-\frac{8 f_{i} f_{i j} f_{j}}{(1-f)^{3}}-\frac{2 f_{i}^{2} f_{j j}}{(1-f)^{3}}-\frac{6 f_{i}^{2} f_{j}^{2}}{(1-f)^{4}} \\
= & \frac{4 f_{j} f_{i j} f_{j}-2 \operatorname{Ric}_{i j} f_{i} f_{j}}{(1-f)^{2}}+\frac{2|\nabla f|^{4}}{(1-f)^{3}}+\frac{2|\nabla f|^{2}\left(D e^{f}\right)^{p-1}}{(1-f)^{2}}\left[(p-1)+\frac{1}{1-f}\right] \\
& -\frac{2 f_{i j}^{2}}{(1-f)^{2}}-\frac{8 f_{i} f_{i j} f_{j}}{(1-f)^{3}}-\frac{6 f_{i}^{2} f_{j}^{2}}{(1-f)^{4}} .
\end{aligned}
$$


As by hypothesis, $f \leq 0$, we have

$$
\left[(p-1)+\frac{1}{1-f}\right] e^{(p-1) f} \leq p,
$$

hence, since $\operatorname{Ric}_{i j} f_{i} f_{j} \geq K(n-1)|\nabla f|^{2}$, we get

$$
\begin{aligned}
w_{t}-\Delta w & \leq \frac{4 f_{i} f_{i j} f_{j}}{(1-f)^{2}}+\frac{2|\nabla f|^{4}}{(1-f)^{3}}+\frac{2\left(p D^{p-1}-(n-1) K\right)|\nabla f|^{2}}{(1-f)^{2}} \\
& -\frac{2 f_{i j}^{2}}{(1-f)^{2}}-\frac{8 f_{i} f_{i j} f_{j}}{(1-f)^{3}}-\frac{6|\nabla f|^{4}}{(1-f)^{4}} .
\end{aligned}
$$

For the sake of simplicity let us set $L=\left(p D^{p-1}-(n-1) K\right)$. Notice that by (3.2), there holds

$$
\langle\nabla f \mid \nabla w\rangle=\frac{2 f_{i} f_{i j} f_{j}}{(1-f)^{2}}+\frac{2|\nabla f|^{4}}{(1-f)^{3}}
$$

hence, substituting, we get

$$
\begin{aligned}
w_{t}-\Delta w & \leq 2\langle\nabla f \mid \nabla w\rangle-\frac{2|\nabla f|^{4}}{(1-f)^{3}}+\frac{2 L|\nabla f|^{2}}{(1-f)^{2}}-\frac{2 f_{i j}^{2}}{(1-f)^{2}}-\frac{8 f_{i} f_{i j} f_{j}}{(1-f)^{3}}-\frac{6|\nabla f|^{4}}{(1-f)^{4}} \\
& =\left(2-\frac{2}{1-f}\right)\langle\nabla f \mid \nabla w\rangle+\frac{2 L|\nabla f|^{2}}{(1-f)^{2}}-\frac{2|\nabla f|^{4}}{(1-f)^{3}}-\frac{2 f_{i j}^{2}}{(1-f)^{2}}-\frac{4 f_{i} f_{i j} f_{j}}{(1-f)^{3}}-\frac{2|\nabla f|^{4}}{(1-f)^{4}} \\
& =-\frac{2 f}{1-f}\langle\nabla f \mid \nabla w\rangle+\frac{2 L|\nabla f|^{2}}{(1-f)^{2}}-\frac{2|\nabla f|^{4}}{(1-f)^{3}}-\frac{2}{(1-f)^{2}}\left(f_{i j}+\frac{f_{i} f_{j}}{1-f}\right)^{2} \\
& \leq-\frac{2 f}{1-f}\langle\nabla f \mid \nabla w\rangle+2 L w-2(1-f) w^{2} .
\end{aligned}
$$

We introduce the following cut-off functions (of Li and Yau [9]). Let $\psi$ be a smooth function supported in $Q_{R, T}$ with the following properties:

1. $\psi(x, t)=\varphi\left(d^{M}\left(x_{0}, x\right), t\right) \in[0,1]$ with $\varphi(r, t) \equiv 1$ if $r \leq R / 2$ and $T_{0}-T / 4 \leq t \leq T_{0}$,

2. $\varphi$ is nonincreasing in the space variable $r$,

3. $|\nabla \psi| / \psi^{a}=\left|\partial_{r} \varphi\right| / \varphi^{a} \leq C_{a} / R$ and $\left|\partial_{r r}^{2} \varphi\right| / \varphi^{a} \leq C_{a} / R^{2}$, when $0<a<1$,

4. $\left|\partial_{t} \psi\right| / \psi^{1 / 2} \leq C / T$,

for some constants $C, C_{a}$ independent of $R$ and $T$. has

Then, by inequality (3.3) with a straightforward calculation, setting $b=-\frac{2 f}{1-f} \nabla f$ one

$$
\begin{aligned}
\Delta(\psi w) & +\langle b \mid \nabla(\psi w)\rangle-2\left\langle\frac{\nabla \psi}{\psi} \mid \nabla(\psi w)\right\rangle-(\psi w)_{t} \\
& \geq 2 \psi(1-f) w^{2}+\langle b \mid \nabla \psi\rangle w-2 \frac{|\nabla \psi|^{2}}{\psi} w+w \Delta \psi-\psi_{t} w-2 L w \psi
\end{aligned}
$$


Suppose that the positive maximum of $\psi w$ is reached at some point $\left(x_{1}, t_{1}\right) \in Q_{R, T}$, which cannot be on the boundary where $\psi=0$. Arguing again (as in Lemma 2.5) in the sense of support function, if necessary, at such maximum point there holds $\Delta(\psi w) \leq 0$, $(\psi w)_{t}=0$ and $\nabla(\psi w)=0$, hence

$$
2 \psi(1-f) w^{2}\left(x_{1}, t_{1}\right) \leq-\left[\langle b \mid \nabla \psi\rangle w-2 \frac{|\nabla \psi|^{2}}{\psi} w+(\Delta \psi) w-\psi_{t} w-2 L w \psi\right]\left(x_{1}, t_{1}\right) .
$$

We now estimate each term on the right hand side. For the first term we have,

$$
\begin{aligned}
|\langle b \mid \nabla \psi\rangle w| & \leq \frac{2|f|}{1-f}|\nabla f||\nabla \psi| w \\
& =2 w^{3 / 2}|f||\nabla \psi| \\
& =2\left[(1-f) \psi w^{2}\right]^{3 / 4} \frac{|f||\nabla \psi|}{[(1-f) \psi]^{3 / 4}} \\
& \leq(1-f) \psi w^{2}+C \frac{(f|\nabla \psi|)^{4}}{[(1-f) \psi]^{3}} \\
& \leq(1-f) \psi w^{2}+C \frac{f^{4}}{R^{4}(1-f)^{3}},
\end{aligned}
$$

by the properties of the function $\psi$.

For the second term,

$$
\frac{|\nabla \psi|^{2}}{\psi} w=\psi^{1 / 2} \frac{|\nabla \psi|^{2}}{\psi^{3 / 2}} w \leq \frac{1}{8} \psi w^{2}+C\left(\frac{|\nabla \psi|^{2}}{\psi^{3 / 2}}\right)^{2} \leq \frac{1}{8} \psi w^{2}+\frac{C}{R^{4}} .
$$

Thanks to the assumption on the nonnegative Ricci curvature, by the Laplacian comparison theorem (see formula (2.1)), we have

$$
\begin{aligned}
-(\Delta \psi) w & \leq-\left[\partial_{r r}^{2} \varphi+\frac{n-1}{r} \partial_{r} \varphi\right] w \\
& \leq\left[\left|\partial_{r r}^{2} \varphi\right|+\frac{2(n-1)}{R}\left|\partial_{r} \varphi\right|\right] w \\
& \leq \varphi^{1 / 2} w\left(\frac{\left|\partial_{r r}^{2} \varphi\right|}{\varphi^{1 / 2}}+2(n-1) \frac{\left|\partial_{r} \varphi\right|}{R \varphi^{1 / 2}}\right) \\
& \leq \frac{1}{8} \varphi w^{2}+C\left(\left[\frac{\left|\partial_{r r}^{2} \varphi\right|}{\varphi^{1 / 2}}\right]^{2}+\left[\frac{\left|\partial_{r} \varphi\right|}{R \varphi^{1 / 2}}\right]^{2}\right) \\
& \leq \frac{1}{8} \psi w^{2}+\frac{C}{R^{4}}
\end{aligned}
$$

by the properties of the functions $\varphi$ (we recall that $\partial_{r} \varphi \leq 0$ ) and $\psi$.

Now we estimate $\left|\psi_{t}\right| w$ as

$$
\left|\psi_{t}\right| w=\psi^{1 / 2} \frac{\left|\psi_{t}\right|}{\psi^{1 / 2}} w \leq \frac{1}{8} \psi w^{2}+C\left(\frac{\left|\psi_{t}\right|}{\psi^{1 / 2}}\right)^{2} \leq \frac{1}{8} \psi w^{2}+\frac{C}{T^{2}},
$$


again by the properties of $\psi$.

Finally, we deal with the last term, there holds

$$
2 L w \psi \leq 2 L_{+} w \psi \leq \frac{1}{8} \psi w^{2}+C L_{+}^{2},
$$

as $\psi \leq 1$.

Substituting estimates (3.5), (3.6), (3.7), (3.8), (3.9) in the right hand side of inequality (3.4), we deduce

$$
2(1-f) \psi w^{2} \leq(1-f) \psi w^{2}+C \frac{f^{4}}{R^{4}(1-f)^{3}}+\frac{1}{2} \psi w^{2}+\frac{C}{R^{4}}+\frac{C}{T^{2}}+C L_{+}^{2} .
$$

Recalling that $f \leq 0$, it follows

$$
\psi w^{2}\left(x_{1}, t_{1}\right) \leq C \frac{f^{4}}{R^{4}(1-f)^{4}}+\frac{1}{2} \psi w^{2}\left(x_{1}, t_{1}\right)+\frac{C}{R^{4}}+\frac{C}{T^{2}}+C L_{+}^{2}
$$

and, since $f^{4} /(1-f)^{4} \leq 1$, we conclude that

$$
\psi^{2}(x, t) w^{2}(x, t) \leq \psi^{2}\left(x_{1}, t_{1}\right) w^{2}\left(x_{1}, t_{1}\right) \leq \psi\left(x_{1}, t_{1}\right) w^{2}\left(x_{1}, t_{1}\right) \leq \frac{C}{R^{4}}+\frac{C}{T^{2}}+C L_{+}^{2},
$$

for all $(x, t) \in Q_{R, T}$.

As $\psi=1$ in $Q_{R / 2, T / 4}, w=|\nabla f|^{2} /(1-f)^{2}$ and $L=\left(p D^{p-1}-(n-1) K\right)$, we finally have

$$
\frac{|\nabla f|}{(1-f)} \leq \frac{C}{R}+\frac{C}{\sqrt{T}}+C \sqrt{\left(p D^{p-1}-(n-1) K\right)_{+}}
$$

for every $(x, t) \in Q_{R / 2, T / 4}$. Since $f=\log (u / D)$, we are done.

The constant $C$ can be effectively traced back and made explicit from estimates (3.5) onward: it comes from reiterated applications of Young's inequality with numerical constants, from the properties 3 and 4 of the cut-off functions $\psi$ through the constants $C_{a}$ and $C$ respectively, and from the constant $2(n-1)$ appearing in the Laplacian comparison theorem used in estimate (3.7). So $C(n, p)$ is depending only on the dimension $n$ of the manifold $M$ and on the exponent $p>1$.

Remark 3.2. Notice that if $K>0$, then the manifold is compact, by Bonnet-Myers theorem (see [7]).

If $u \leq D$ uniformly in $M \times\left[T_{0}-T, T_{0}\right]$, then estimate (3.1) holds for every $(x, t) \in$ $M \times\left[T_{0}-T / 4, T_{0}\right]$, when $R$ is large enough, hence, sending $R \rightarrow+\infty$, we get the following corollary.

Corollary 3.3. Let $(M, g)$ be an $n$-dimensional Riemannian manifold such that $\mathrm{Ric} \geq K(n-1)$, for some $K \geq 0$. Let $u$ be a positive solution of the semilinear heat equation $u_{t}=\Delta u+|u|^{p}$ in $M \times\left[T_{0}-T, T_{0}\right]$, for $p>1$. Assume that $u \leq D$, then, there exists $C=C(n, p)$ such that

$$
\frac{|\nabla u(x, t)|}{u(x, t)} \leq C\left(\frac{1}{\sqrt{T}}+\sqrt{\left(p D^{p-1}-(n-1) K\right)_{+}}\right)\left(1+\log \frac{D}{u(x, t)}\right)
$$

for every $(x, t)$ in $M \times\left[T_{0}-T / 4, T_{0}\right]$. 
Remark 3.4. If $M$ is compact, one can also prove this corollary directly following the proof of Lemma 3.1, simply considering functions $\psi$ which are constant in space.

Corollary 3.5. Let $(M, g)$ be an n-dimensional Riemannian manifold such that Ric $\geq K(n-1)$, for some $K \geq 0$ and let $p>1$. Let $u$ be a nonzero ancient solution of the semilinear heat equation $u_{t}=\Delta u+|u|^{p}$ in $M \times\left(-\infty, T_{0}\right]$ bounded by $D>0$. Then, there exists a constant $C=C(n, p)$ such that for every $(x, t) \in M \times\left(-\infty, T_{0}\right]$ there holds

$$
\frac{|\nabla u(x, t)|}{u(x, t)} \leq C \sqrt{\left(p D^{p-1}-(n-1) K\right)_{+}}\left(1+\log \frac{D}{u(x, t)}\right),
$$

where $\left(p D^{p-1}-(n-1) K\right)_{+}$denotes $\max \left\{p D^{p-1}-(n-1) K, 0\right\}$.

Proof. By Theorem 2.6, we know that if $u$ is nonzero then $u$ is necessarily positive. Then, since estimate (3.1) holds for every $(x, t) \in M \times\left(-\infty, T_{0}\right]$, for $R$ and $T$ large enough, sending $R, T \rightarrow+\infty$, we get the conclusion.

Following Poláčik-Quittner-Souplet [5] for the Euclidean case, we now prove a universal a priori estimate for ancient solutions of $u_{t}=\Delta u+|u|^{p}$, with $1<p<\frac{n(n+2)}{(n-1)^{2}}$ on an $n$-dimensional Riemannian manifold $(M, g)$ with bounded geometry (injectivity radius positively uniformly bounded below and uniformly bounded Riemann tensor with all its covariant derivatives), for instance when $M$ is compact. As a corollary, such ancient solutions decay to zero at minus infinity.

Proposition 3.6. Let $(M, g)$ be an n-dimensional Riemannian manifold with bounded geometry. Let $u$ be a uniformly bounded below solution of the equation $u_{t}=\Delta u+|u|^{p}$ in $M \times\left(T_{0}, T\right)$, with $1<p<\frac{n(n+2)}{(n-1)^{2}}$. Then there exists a universal constant $C=C(n, p)$ such that there holds

$$
u(x, t)+|\nabla u(x, t)|^{\frac{2}{p+1}} \leq C\left[\left|t-T_{0}\right|^{-\frac{1}{p-1}}+|T-t|^{-\frac{1}{p-1}}\right],
$$

for every $(x, t) \in M \times\left(T_{0}, T\right)$.

Proof (Sketch). One can argue precisely in the same way as in the proof of Theorem 3.1 (ii) in [5], concerning the case $M=\mathbb{R}^{n}$. In such paper, assuming the conclusion fails, by a blow-up argument the authors can produce an eternal, nonzero, bounded and positive (classical) solution of equation $u_{t}=\Delta u+u^{p}$ in the whole $\mathbb{R}^{n}$, which is known it does not exist (see Theorem A in the same paper). In our case of a manifold, the only difference in taking such blow-up (which is given by an appropriate "rescaling" of the solution) is that we have to "rescale" (dilate) also the ambient space $(M, g)$. Anyway, by the hypothesis of bounded Riemann tensor and injectivity radius uniformly bounded below, the dilated manifolds (together with the rescaled functions defined on them) smoothly converge, up to a subsequence, in the sense of Cheeger-Gromov (see [13], for instance) to $\mathbb{R}^{n}$ with its canonical flat metric. Hence, as for the case $M=\mathbb{R}^{n}$ discussed in [5], we obtain a nonnegative solution $u_{t}=\Delta u+u^{p}$ in the whole $\mathbb{R}^{n}$, with the same properties as above, which is a contradiction. 
As a corollary, sending $T_{0} \rightarrow-\infty$, we have the following decay estimate for ancient solutions, which must be nonnegative (hence bounded below) by Theorem 2.6.

Corollary 3.7. Let $(M, g)$ be an $n$-dimensional Riemannian manifold with bounded geometry. Let $u$ be an ancient solution of the equation $u_{t}=\Delta u+|u|^{p}$ in $M \times(-\infty, T)$, with $1<p<\frac{n(n+2)}{(n-1)^{2}}$, for some $T \in \mathbb{R}$. Then there exists a universal constant $C=C(n, p)$ such that

$$
u(x, t) \leq C(T-t)^{-\frac{1}{p-1}} \text { for any }(x, t) \in M \times(-\infty, T) .
$$

In particular

$$
\lim _{t \rightarrow-\infty} \max _{x \in M} u(x, t)=0 .
$$

If the solution is actually eternal, we can also send $T \rightarrow+\infty$, concluding that $u \equiv 0$.

Corollary 3.8. Let $(M, g)$ be a n-dimensional Riemannian manifold with bounded geometry. Every eternal solution of the equation $u_{t}=\Delta u+|u|^{p}$ in $M \times \mathbb{R}$, with $1<p<\frac{n(n+2)}{(n-1)^{2}}$, is identically zero.

Remark 3.9. The conclusion of Proposition 3.6, hence also of Corollaries 3.7 and 3.8, is conjectured to hold for the whole range of exponents $p \in\left(1, \frac{n+2}{n-2}\right)$, if $n \geq 3$, or $p \in(1,+\infty)$, when $n=1,2$. This would follow by the nonexistence of eternal, positive, classical solutions of equation $u_{t}=\Delta u+u^{p}$ in the whole $\mathbb{R}^{n}$, which at the moment can be shown in general only when $p<\frac{n(n+2)}{(n-1)^{2}}$ and assuming that such solutions are radial when $p \in$ $\left[\frac{n(n+2)}{(n-1)^{2}}, \frac{n+2}{n-2}\right)$ (see the discussion in [5, Section 2] and the references therein).

\section{Triviality}

We can now prove a triviality result and, consequently, Theorem 1.2 stated in the introduction.

Theorem 4.1. Let $(M, g)$ be an $n$-dimensional compact Riemannian manifold such that Ric $>0$. Let $u$ be an ancient solution to the semilinear heat equation $u_{t}=\Delta u+|u|^{p}$, with $p>1$, such that

$$
\lim _{t \rightarrow-\infty} \max _{x \in M} u(x, t)=0,
$$

then $u$ is trivial.

Proof. As the Ricci tensor is positive and $M$ compact, Ric $\geq(n-1) K g$, for some $K>0$. By Theorem 2.6 we then know that, if $u$ is nonzero, then $u$ is necessarily positive. Under the above growth hypothesis, there exists $T_{0} \in \mathbb{R}$ such that

$$
0<u(x, t) \leq[(n-1) K / p]^{\frac{1}{p-1}}
$$


for every $x \in M$ and $t \leq T_{0}$, hence, by the estimate (3.10), we get (with a constant $C$ depending only on the dimension $n$ of the manifold $M$ and on the exponent $p>1$ ),

$$
\frac{|\nabla u(x, t)|}{u(x, t)} \leq C \sqrt{\left(p D^{p-1}-(n-1) K\right)_{+}}\left(1+\log \frac{D}{u(x, t)}\right)=0,
$$

for every $(x, t) \in M \times\left(-\infty, T_{0}\right]$, as $D=\max _{M \times\left(-\infty, T_{0}\right]} u(x, t) \leq[(n-1) K / p]^{\frac{1}{p-1}}$.

Being $|\nabla u(x, t)|=0$ for every $(x, t) \in M \times\left(-\infty, T_{0}\right]$, the function $u$ is constant in space for every $t \leq T_{0}$. By uniqueness of solutions ( $M$ is compact), $u$ is trivial.

Remark 4.2. The hypothesis (4.1) can be slightly weakened as follows. If Ric $\geq(n-1) K g$, it is sufficient that

$$
\limsup _{t \rightarrow-\infty} \max _{x \in M} u(x, t)<[(n-1) K / p]^{\frac{1}{p-1}} .
$$

Proof of Theorem 1.2. The conclusion follows immediately by putting together Theorem 4.1 with the decay estimate of Corollary 3.7.

\section{References}

[1] T. Aubin and Y.-Y. Li, On the best Sobolev inequality, J. Math. Pures Appl. (9) 78 (1999), no. 4, 353-387.

[2] D. Castorina and C. Mantegazza, Ancient solutions of semilinear heat equations on Riemannian manifolds, Atti Accad. Naz. Lincei Rend. Lincei Mat. Appl. 28 (2017), no. 1, 85-101.

[3] B.-L. Chen, Strong uniqueness of the Ricci flow, J. Diff. Geom. 82 (2009), 363-382.

[4] P. Poláčik, P. Quittner, and P. Souplet, Singularity and decay estimates in superlinear problems via Liouville-type theorems. I. Elliptic equations and systems, Duke Math. J. 139 (2007), no. 3, 555-579.

[5] __ Singularity and decay estimates in superlinear problems via Liouville-type theorems. II. Parabolic equations, Indiana Univ. Math. J. 56 (2007), no. 2, 879-908.

[6] M. G. Crandall, H. Ishii, and P.-L. Lions, User's guide to viscosity solutions of second order partial differential equations, Bull. Amer. Math. Soc. 27/1 (1992), 1-67.

[7] S. Gallot, D. Hulin, and J. Lafontaine, Riemannian geometry, Springer-Verlag, 1990.

[8] R. S. Hamilton, Four-manifolds with positive curvature operator, J. Diff. Geom. 24 (1986), no. $2,153-179$.

[9] P. Li and S.-T. Yau, On the parabolic kernel of the Schrödinger operator, Acta Math. 156 (1986), no. 3-4, 153-201. 
[10] C. Mantegazza, Lecture notes on mean curvature flow, Progress in Mathematics, vol. 290, Birkhäuser/Springer Basel AG, Basel, 2011.

[11] C. Mantegazza, G. Mascellani, and G. Uraltsev, On the distributional Hessian of the distance function, Pacific J. Math. 270 (2014), no. 1, 151-166.

[12] F. Merle and H. Zaag, Optimal estimates for blowup rate and behavior for nonlinear heat equations, Comm. Pure Appl. Math. 51 (1998), no. 2, 139-196.

[13] P. Petersen, Riemannian geometry, second ed., Graduate Texts in Mathematics, vol. 171, Springer, New York, 2006.

[14] P. Quittner, Liouville theorems for scaling invariant superlinear parabolic problems with gradient structure, Math. Ann. 364 (2016), no. 1-2, 269-292.

[15] W. Sheng, Laplacian, volume and Hessian comparison theorems, http://www.math.zju.edu.cn/swm/RG_Section_6.pdf, 2009.

[16] P. Souplet and Q. S. Zhang, Sharp gradient estimate and Yau's Liouville theorem for the heat equation on noncompact manifolds, Bull. London Math. Soc. 38 (2006), no. 6, 10451053.

[17] G. Talenti, Best constant in Sobolev inequality, Ann. Mat. Pura Appl. (4) 110 (1976), 353-372. 